\title{
Las proyecciones del Trabajo Social contemporáneo: una observación desde la Teoría de los Sistemas Sociales
}

The Projections of Contemporary Social Work: An Observation from the Theory of Social Systems

Felipe Rivera Silva

Investigador en el Departamento de Educación de Fundación Integra (Región del Bíobio, Chile). Sociólogo, Universidad de Concepción (Chile). Alumno del Magister en Análisis Sistémico aplicado a la Sociedad (MaSS), Universidad de Chile. Diagonal Pedro Aguirre Cerda \# 1225, Concepción, Chile - Código Postal: 4030000. Correo electrónico: felirivera@ug.uchile.c.

Esteban Cofré Morales

Investigador en SENDA - Previene, Peñalolén (Región Metropolitana, Chile). Trabajador Social, Universidad Alberto Hurtado (Chile). Alumno del Magister en Anólisis Sistémico aplicado a la Sociedad (MaSS). Universidad de Chile Dirección Postal Instatucional: Calle Llanura \# 6325, Lo Hermida, Peñalolén, Chile - Código Postal: 7910000. Correo electrónico: ecofre@ug.uchile.cl

\section{Resumen}

El presente artículo se orienta a establecer una aproximación desde la Teoría de los Sistemas Sociales desarrollada por Niklas Luhmann hacia los fundamentos del Trabajo Social contemporáneo. De acuerdo a lo anterior, se elabora un abordaje de las principales directrices que comprende tal disciplina en la actualidad, al mismo tiempo que se despliegan los recursos teóricos y conceptuales más atingentes de la teoria elaborada por Luhmann para la realización de este análisis. A la luz de ambos niveles, se articula una propuesta que arroja luces acerca del Trabajo Social como un sistema funcional de la sociedad moderna, para luego terminar con un apartado que recoge los alcances más consustanciales e interrogantes que quedan abiertas para futuras investigaciones. Palabras clave. Trabajo Social contemporáneo, Teoría de los Sistemas Sociales, Intervención Social, Domicilio Social.

\section{Abstract}

The present article aims to establish an approchement to the foundations of contemporary socia work from the Theory of Social Systems developed by Niklas Luhmann. According to this, the main guidelines of this discipline and the most impotant theoretical and conceptual resources of the theory developed by Luhmann are deployed. From both levels, a proposal that sheds light on socia work as a functional system of modern society is articulated; ending with a section that shows the most important questions and scopes that remain open for future research.

Key words. Contemporary Social Work, Social Systems Theory, Social practice, Social Address

\section{Introducción}

Desde los principales ámbitos ligados al devenir contingente de la modernidad, la Teoría de los Sistemas Sociales se ha logrado imbricar como un entramado conceptual gravitante en los círculos académicos más heterogéneos. A partir de esto, su relevancia ha estado ampliamente ligada con las posibilidades que brinda de enarbolar esquemas de observación acerca de la complejidad y autodescripción inherente a la sociedad contemporánea (Luhmann, 1998; 2007), recurso que tiene la particularidad de abordar todos los ámbitos operativos que convergen en el seno de esta última. En tal sentido, el Trabajo Social se ha convertido, paulatinamente, en uno de los focos de atención más destacados en las investigaciones que se abanderan con la perspectiva de los Sistemas Sociales. De hecho, hay quienes indican que esta disciplina se ha convertido -actualmente- en el tema predilecto de los autores sistémicos (Robles, 2010), dados sus alcances respecto a la actualización de los eventos comunicativos vinculados al metacódigo inclusión/exclusión en la sociedad contemporánea.

En vista de dichas nociones, es necesario agregar 
que uno de los principales argumentos que subyacen a este interés en las bases operativas del Trabajo Social, responde a sus atribuciones como sistema funcional, lo que dicho en términos concretos, hace alusión a cómo opera - de forma altamente especializada- median gias que mermen la irrestricta exclusión a la que se ven enfrentados cotidianamente los casos - tanto sujetos individualizados como grupos, familias y/o comunidades- que son atendidos por los(as) trabajadores(as) sociales. Desde tales consideraciones, la formación académica del Trabajo Socia -desde la perspectiva de los Sistemas Sociales- se alza igualmente como una temática a profunizar, ya que se pone acento en que esta disciplina se enLo realmente significativo de tal condición, se pone de manifiesto en las atribuciones exclusivamente praxeológicas que ha recibido el Trabajo Social, en función de lo cual, sus intervenciones son vistas -de forma sesgada, dicho sea de paso- como un mecanismo que pretr por los altos niveles de exclusión que concretiza en la forma de domicilios sociales dañados.

En base a lo anterior, nuestra propuesta está orientada a recoger los alcances operativos del Trabajo Social desde la acera opuesta, es decir, desde un enfoque sistémico que logre observar su rol académico y profesional en torno a la actualización del código inclusión/exclusión, y en cómo, a través de esto, logra incidir en el fomento de domicilios sociales auspiciosos y en una maximización de la forma persona en la sociedad contemporánea.

\section{Lineamientos axiales: los fundamentos}

\section{del Trabajo Social contemporáneo}

Desde la segunda mitad del siglo pasado, el Trabajo Social ha evidenciado un abordaje intrínsecamente reflexivo en torno a los principales lineamientos que se hallan vinculados a su quehacer profesional, como también, a sus entramados operativos como disciplina científica que se ha forjado mediante encuentro de los elementos históricos, sociales, culturales y sim bl cos que han caractenzado el deven contingente de la sociedad moderna. En función de esto, las representaciones contemporáneas del Trabajo Social son indispensables para desmantelar las formas en que la modernidad ha plasmado esquemas de análisis generales acerca de las ideas, perspectivas

y enfoques que orientan los marcos de referencia acerca de la(s) realidad(es) social(es) imbricada(s) al acontecer recursivo de la sociedad actual.

Eventualmente, el Trabajo Social contemporáneo debió superar una lógica desarrollista y paliativa que buscaba asociar a esta disciplina con una concepción asistencialista, como también, con un recurso instrumental para la reproducción del sistema dominante (Quezada, et al, 2002). En virtud de esto, se comenzó a elaborar una reconceptualización de los fundamentos relativos al Trabajo Social, lo que tuvo como objetivo esencial el "producir un cancir un referente al marco teórico vigente hasta ese momento en la profesión" (Ander-Egg, 1984, p.35). Lo cierto es que tales alcances llevaron a que esta disciplina asumiera perspectivas disidentes a la influencia capitalista en las sociedades latinoamericanas, lo que posibilitó identificar desde posturas que desontologizaban el concepto de progreso y desarrollo al interior del Trabajo Social, hasta visiones que lo comprendían como una herramienta al servicio de la resistencia popular (Healy, 2001).

En este punto, es factible encontrar una serie de aportes cruciales para la presente revisión. Uno de los más destacados, se encuentra relacionado a la postura de Vicente de Paula Faleiros (1970), quien realizó un minucioso estudio acerca de cuáles eran las bases ideológicas y me las bases ideologicas y metodologicas que se le atribuían en aquel periodo al Trabajo Social, teniendo como punto de referencia las actas de sesiones que fueron elaboradas a lo largo de los primeros congresos que se desarrollaron en países latinoamericanos tales como Chile, Perú, Costa Rica, por nombrar algunos. Por su parte, el autor da cuenta, a lo largo de su revisión, de los motivos por los cuales urgen iniciativas críticas sobre los alcances de la intervención social (en cuanto a sus fundamentos e ideologías), a raíz de lo cual, elabora una serie de aportes desde la reconceptualización de las Ciencias Sociales, los que desembocaron principalmente en una observación de la realidad ética y política que comprende gran parte de América Latina ${ }^{1}$.

Posteriormente, durante los años 80 se constataron grandes aportes desde el trabajo de Minuchin y Charles (1984), quienes se centraron en los alcances operativos que daba cuenta el Trabajo Social con relación al área de la Terapia Familiar. Sin embargo, no fue hasta la mitad de los años 90 que la disciplina encontró una reconceptualización basada en la necesidad de construir categorías de análisis que permitieran mostrar una realidad persistente y múltiple. En palabras de Matus (1999), el Trabajo Social se articula mediante "las posibilida政 [que] requieren de una adecuada comprensión del contexto. De otro modo, solo se acentuará la separación entre interpretación e intervención. Con una interpretación encapsulada, se genera una intervención débil o estrictamente funcional, donde queda imposibilitado el trabajo del concepto. Las transformaciones también" (p. 18). En función de

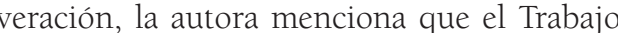
Social debe ser abordado mediante dos nociones: la primera de ellas se orienta a reflejar que la concepción tecnológica del Trabajo Social -en sus diversas vertientes- se ha vuelto claramente insuficiente y problemática, lo que responde a que los desafíos de la globalización no pueden ser resueltos mediante la utilización de nuevas tecnologías que reemplacen el rol litigante de ajadores(as) sociales, ya que "los conceptos y modelos de la intervención social $[. .$.$] aparecen volcados hacia una noción de$ acción práctica, enfatizando la dimensión espacial (el terreno, el lugar, el campo) donde se ejerce el quehacer profesional, intentando dar un buen servicio a la gente. De allí que incluso se llegue a decir, que este saber se desarrolla cara a cara, variando el número de personas involucradas según se trate de una atención de caso, de grupos o de comunidades" (Matus, 2003, p.1)

Mientras que la segunda noción, dice relación con establecer una dialéctica entre la teoría y la praxis al interior del Trabajo Social, con la finalidad de elaborar una aproximación efectiva a la complejidad de la intervención en el contexto de la sociedad contemporánea. De acuerdo a esto, la formación profesional de los(as) trabajadores(as) sociales comprende revelar que los sujeto-objetos no pueden -ni deben- ser entendidos bajo la idea única de un caso no individualizado, ni tampoco como un público generalizado al que se debe atender. En consecuencia, nadie llega en su condición de persona natural a solicitar los servicios de un(a) trabajador(as) social, estegoría analítica determinada: mujer golpeada, cesante, menor en situación irregular, directiva de una organización sindical, integrantes de un campamento (Matus, 2003). Con todo y lo anterior, la propuesta contemporánea del Trabajo Social articulada por Matus (1999), fija su atención en cuatro fundamentos básicos que dan origen a una Intervención Social Fundada. A par- tir de esta última, la autora precisa que existe una relación mediada insustituible entre intervención y un sistema de comprensión social constituido a menos por cuatro dimensiones relacionadas aunque no homologables: los cambios existentes en el contexto, las diversas perspectivas de teorías sociales, los enfoques epistemológicos y los marcos ético/valóricos" (p. 27). Tales aspectos extienden la posibilidad de observar cómo la resignificación disciplinar del Trabajo Social, se alza desde la crítica a las formas clásicas de acción insertas en la intervención social, dando paso a una interpretación de esta última desde su función mediadora. En otras pala bras, se busca destacar que la función de mediación "involucra no un procedimiento de bisagras, sino la posibilidad de nombrar un escenario posible de reconstrucciones, una tarea. La función de mediación no es hablar de otros, sino mostrar las contradicciones de los discursos" (Quezada, et al, 2002).

Esquemas primigenios: los dominios de la teoría de los Sistemas Sociales

A partir de lo anterior, la complejidad estructural de estos alcances permite establecer un diálogo con los ejes que cautelan la forma y estabilidad de los postulados desarrollados por Niklas Luhmann, con especial atención en la relevancia que este le atribuye a la sociedad moderna y a los principios de diferenciación a los que se encuentra supeditada. En orden a establecer un punto de origen, es necesario mencionar que tales premisas se encuentran ligadas -indefectiblemente- con las disposiciones estructurales que la sociedad moderna configura para establecer sus propios límites operativos (Luhmann, 1992), es decir, los esquemas recursivos de comunicación que al interior de la sociedad moderna se encuentran altamente especializados, y que en última instancia, se orientan a impregnar de reflexividad todo lo que se encuentra bajo su alero.

Luego, existe la posibilidad de mencionar que la comunicación -entendida como la unidad básica de lo social desde la visión teórica de Luhmannestá vinculada a esferas muy diversas al interior de la sociedad contemporánea. Dentro de este marco, ámbitos tales como la política, la economía, la religión, la familia y la educación -por nombra algunos-, pueden ser definidos como sistemas de comunicación que tienen la particularidad de desplegar funciones parceladas (Luhmann, 1996). Por consiguiente, la independencia de cada uno de esto sistemas se remite a un principio argumentativo ligado a la actualización constante de sus operaciones, mediante lo cual se originan procesos determinados 
internamente por los propios sistemas.

En virtud de tales consideraciones, los sistemas aíslan cualquier vinculación complementaria con elementos externos a sí mismos, o alguna determinación exógena que altere su estructura operat Tal condición demanda sopesar que los sistemas, además de repeler cualquier alteración ajena a sus propia autoorganización, deben recurrir a operaciones específicas para lograr mantener vigente su hiperautonomía. Atendiendo a estas consideraciones, Luhmann (2007) sostiene que la fragmentación rrestricta de los sistemas en términos de los alcances que conlleva su selectividad operativa, nombre de diferenciación funcional. En lo esencial las estructuras jerárquicas de los procesos comunicativos con que operan los sistemas, se encuentran diferenciadas por las funciones específicas que cada uno de estos lleva a cabo. Ahora bien, lo realmente significativo de esto dice relación con el modo en que la diferenciación funcional se encuentra ligada la evolución socioculuraly aumento de lacomplejilad estrucural que presenta la modernict.

De allí que sea perentorio relacionar a la sociedad moderna con una noción descentralizada de los sistemas funcionales, lo que a su vez repercute en el hecho de evitar cualquier precepto que abone favor de una inclusión y exclusión uniforme. De este modo, ambos conceptos deben ser entendidos como la realización de una operación basada en un criterio dual y autoexcluyente.

En este sentido, tanto la exclusión como la inclusión fueron abordadas por Luhmann (1998) a partir de sustitución que hace de la noción de "integración" desarrollada por Talcott Parsons (1988), la que atribuía una "orientación normativa a la inclusión desie el papel quet tación de los individuos, con lo cual estos pasaban de ser aceptados con un estatus determinado en el sistema social, a ser incluidos en dicho sistema, y por lo tanto, integrados a él" (Robles, 2005, p.19). Frente a tales aspectos, Luhmann considera que el gran inconveniente de lo expuesto por su maestro, radica en que su gran interés por los comportamientos gentó que la exclusión se considerada como una desviación repelida por la integración, lo que generó que se invisibilizarla por completo en el esquema conceptual de Parsons.

Por lo mismo, Luhmann (2007) se inclina por describir a la inclusión y exclusión como ejes inseparables desde el ámbito de la desigualdad social en funcion de la cual pretendía describir el modo en que la so- ciedad contemporánea regula la participación de la forma persona, al igual que su comportamiento frente a terceros (Luhmann, 1998). Al instante de mencionar a la forma persona, no se apunta a su tematización ontológica 0 a su abordaje como ente trascendental, sino que a su observacion ación como artefacto comunicativo que opera mediante biografías individuales actualizadas en base a los requerimientos que los sistemas funcionales exigen para que sean incluidos en las prestaciones auspiciosas de sus funciones (Robles, 2002). Según esto, la sociedad contemporánea distingue entre personas y no personas, mientras que los sistemas funcionales operan como catalizadores dotados de posibilidades de inde inclusión, pero también como promotores de fuertes mecanismos de exclusión. En tal sintonía, la base de la forma persona alude a un perfil de cómo se ordenan nuestras vidas en función del interruptor de la inclusión/exclusión, el que está presente en ámbitos tan distintos como un club deportivo o las relaciones de pareja. El resultado de la inclusión es ser tratado como persona, que en términos concretos, alude a terer acceso a las prestaciones más beneficiosas de los sistemas funcionales. Mientras, que por el contrario, el ser excluido y tratado como no persona, se refleja en los casos de los enfermos mentales, presidiarios, portadores de algún estigma, entre otros.

En consecuencia, las aristas de la forma persona evidencian una proyección comunicativa en torno a uno de los conceptos más imbricados en la diferenciación funcional de la sociedad moderna: el de domiciliaridad social. Por intermedio de este recurso teórico, nos referiremos a la domiciliaridad social como el registro de todo nuestro historial individual, al mismo tiempo que a la manera en que este se ha perfilado en relación al código de la inclusión/exclusión. Por ende, al bloquearse el acceso a las prestaciones -entiéndase, beneficios y/o regalías- que se desprenden de las operaciones particulares de los sistemas funcionales, hablaremos de domiciliaridades sociales dañadas, o estructuras comunicativas deterioradas. De este modo, la matriz operativa de la domiciliaridad social se convierte en un acorazado que enarbola las nociones en torno a la "masificación creciente y sostenidu de la exclusión, sumando aquellas como las raciales y étnicas, las generadas como efecto colateral de guerras, las de género, las de trasgresión como la homosexualidad, las exclusiones territoriales, etc." (Robles, 2010, p.132). A pesar de esto, subyacen exigencias de in- clusión a toda costa, sin que con esto se amplíen las posibilidades de su realización. He aquí la jugarreta más malévola de la diferenciación funcional.

Visto de esta manera, el esquema de inclusión/exclusión se ve reflejado bajo la idea de un código binario, el que corresponde a la instrucción basal de sus operaciones. Así, las propiedades del código no permiten valores intermedios ${ }^{3}$-es blanco o negro, sin que se acepten grises- (Luhmann, 1997). Análogamente, al hablar del código binario de la inclusión/exclusión, existe la posibilidad de replicar tales alcances en torno a valores positivos (t) y negativos (-), respectivamente. Con esto, se vulve imprescin nicaciones que irriten a los sistemas funcionales y se codifiquen positivamente, serán procesadas e incluidas por los sistemas, mientras las negativas, serán repelidas y excluidas por los mismos (Luhmann, 2007). Las proyecciones de tal ordenamiento alcanzan a tener gran relevancia en las cualidades dinámicas y estáticas de los sistemas, llegando al punto de moldear el vínculo que existe entre el devenir de las sociedades modernas y su relación con la complejidad estructural que las agobia incesantemente (Robles, 2006a). Tal devenir nos permite, en orden a ilustrar la evolución de la sociedad moderna en torno al código inclusión/exclusión, indicar los estadios previos que existieron antes de fungirse tal cual la conocemos hoy. Estos modelos de sociedades precedentes a la sociedad moderna, y sus respectivas disposiciones operativas frente al código inclusión/exclusión, correspondieron a:

a. La sociedad segmentada: en este ámbito, los matices del código de inclusión/exclusión se expresaron en que la diferenciación de la inserción de los individuos a un segmento determinado consideraba dos ejes fundamentales: la pertenencia a comunidades de residencia o descendencia. Por el contrario, la exclusión de algún individuo respecto a un segmento, se transforma en sinónimo de riesgo inminente, debido a que la interior de dicha organ vincularse hace altamente improbable llegar a sobrevivir de forma aislada. De acuerdo a esto, Makarovic ${ }^{`}$ (2001) sostiene que la división relativa a unidades autosuficientes sin un principio de relaciones jerárquicas -en función de las cuales no existe la necesidad de establecer límites- se alza como el fundamento de sociedades en que la membresía otorgada a partir de la inclusión en un determinado segmento contempla la atribución a niveles que las comunidades usualmente definen en términos de su larga perduración (residir en aldeas o ciudades), basándose en un representación simbólica de la descet (por ejemplo, familias)

b. La sociedad centro/periferia: en este esquema, la diferenciación se basa en fronteras territoriales entre la ciudad y el campo. En tal sentido, su diferenciación emerge como resultado de la expansión que los grandes imperios efectuaron en el contexto de las sociedades segmentadas. En base a esto, su forma de reproducción se vincula con la actualización de la distinción sistema/ entono, con lo cual lo que se indica como centro (sistema) o periferia (entorno) va a depender de representaciones topográficas (Robles, 2010).

c. La sociedad estratificada: en este caso, el interruptor de la inclusión/exclusión se mueve de forma transversal a los estratos sociales. De allí que la inclusión absorba el lado positivo (+) y deseche el lado negativo (-) a partir de la pertenencia a un segmento específico de la población, o como riente, en torno a las castas. En virtud de lo anterior, el pertenecer a un determinado estrato se vincula directamente con el hecho de proveni de una familia en especial, lo que descarta que la inclusión sea un atributo posible de liberar de la descendencia. Por el contrario, el resultar excluido de una familia involucra la directa exclusión de un estrato social en específico. No obstante, el apartarse de un seno familiar no significa necesariamente pasar a incluirse en otro (el caso de matrimonio), pero puede considerar la exclusión de la sociedad, especialmente, si concierne a quienes viven sin lazos familiares efectivos ( $\mathrm{Lu}$ hmann, 1998; Braeckman, 2006). A la luz de las características que revisten la diferenciación en sociedades segmentadas y estratificadas, es posible establecer que en ambas, las fronteras en torno al código inclusión/exclusión se condicen con un principio elemental de inclusión o exclusión total, es decir: un individuo puede estar completamente incluido o excluido de un sistema social (familia o estado), sin posibilidad de ubicarse en una condición intermedia (Luhmann, 2007).

A pesar de esto, el ubicarse en alguno de los lados de la distinción inclusión/exclusión, con acento en 
la diferenciación funcional de la sociedad moderna, está prácticamente determinado por la visibilidad que se alcance en la comunicación de sistemas funcionales tales como la política, la economía, el arte, la familia la educación, entre otros (Robles, 2010). De tal modo, (la permanente ininteligibilidad que determina a tales sistemas, establece la imposibilidad para los individuos de legar a estar completamente incluidos o excluidos. Lo anterior puede ser reflejado de la siguiente manera: En la actualidad es ampliamente legitimado que se pueda participar como votante, consumidor, investigador y amante del arte en condiciones en que se petrenece al sistema politico, al sistema económi econormico-financiero, al sistema de la ciencia al sistema del arte de forma simultánea. Esto deja de manifiesto que es imposible asignar un sistema funcional único para todos los ámbitos de nuestras vidas, debido a que el código inclusión/exclusión prioriza una amplia gama de operaciones sincronizadas. Aún así, estar incluido en uno de estos sistemas, no implica estar incluido en otro de forthas consolidada. Por ejemplo, el estar inclido en el sistema del trabajo, no asegura bajo ningún principio estarlo en el sistema bancario o en de la salud. De hecho, el interruptor inclusión/exclusión opera de tal manera que se hace uso de programas, entendidos como aquellos que "regulan los requisitos de aceptación en el valor positivo (+), y por lo tanto a inclusión, y rechaz exclusión" (Robles, 2006b, p.96). A partir de lo señalado, se evidencia que la inclusión y exclusión operan como un metacódigo que cruz todos -sin excepción- los sistemas funcionales de la sociedad. Sin embargo, en la sociedad contemporánea existe una masificación creciente y sostenida de la exclusión, especialmente por condiciones raciales, étnicas, de género, sexuales, entre otras. Por lo mismo, la exclusión es altamente probable, estable y acumulativa

Desde tal perspectiva, la exclusión se presenta en dos niveles:

a. Exclusión primaria: la que se entiende como el hecho de no tener acceso a las prestaciones que los sistemas funcionales definen como más auspiciosas en términos de servicios elementales, es decir, un trabajo estable, educación completa, salud, protección judicial, familia protectora, etc

b. Exclusión secundaria: la que apunta al hecho de no tener acceso a las redes de influencia que proporcionan beneficios a partir de los generados por los sistemas funcionales, teniendo su base en motivos de limitaciones estructurales -estar recluido en su centro penitenciario u hospital psiquiátrico- o voluntariamente -autoexcluirse o apartarse con pleno conocimiento- (Robles, 2006b)

Mientras que, por otro lado, la inclusión es lábil, improbable y restrictiva, pudiendo dividirse en:

a. Inclusión primaria: la que se entiende como un vasto y amplio acceso a las prestaciones de los sistemas funcionales de la sociedad, lo cual significa llegar a tener u rol protagónico en la cosignifica llegar a tener u rol protagónico en la co-
municación sistémica gracias a dichos beneficios.

b. Inclusión secundaria: aquella que se entiende como el acceso a mundos de la vida excluidos y a las experiencias propias que, a pesar de estar más inclinadas hacia el lado de inclusión, han tenido contacto directo con el lado de la exclusión (Robles, 2004).

A la luz de ambos niveles, es necesario precisar que no existe cabida para una semántica oficial e institucionalizada de la inclusión en la sociedad contempoŕnea, lo que en definitiva conduce (n) a evaluar si las nociones de 'igualdad' y 'equidad' apuntan a invisibilizar la exclusión -como se señaló en apartados anteriores- para subsumirla en el ideario de la inclusión.

\section{Enlace convergente 1: los alcances}

\section{sistémicos del Trabajo Social}

En términos generales, ha existido una propensión histórica a ilustrar el concepto de sistema bajo la forma de una serie definible de elementos que son viables de pormenorizar bajo la clasificación de unidad. Mediante lo anterior, es factible homologar dichos alcances con los lineamientos acuñados por von Bertalunffy (1968) para su Teoria General de Sistemas, en donde menciona que un sistema puede ser definido como una totalidad compuesta por una serie de partes que interactúan entre sí, lo que en última instancia, configura su emplazamiento como entidad.

Desde esta consideración, la visión sistémica clásica se ha enarbolado como uno de los principales marcos de análisis que utilizó el Trabajo Social, amén de las herramientas que brindaba para comprender las dinámicas familiares y las estructuras jerárquicas que se circunscriben a las responsabilidades parentales, recurriendo para esto al paradigma todo/ partes y al perfil de los sistemas dinámicos (Laszlo \& Krippner 1998). No obstante, el desarrollo de nuevas perspectivas sistémicas derivó en la incorporación de nociones aún más específicas y detalladas, entre las que destacó el de los sistemas autorreferenciales. En tal línea, dichos sistemas consolidan su estabilidad mediante el establecimiento de una relación antagónica con sus entornos, en función de la cual se imputa al sistema lo que le es propio, y se relega al entorno aquello que no lo es.

A partir de dichos alcances, la autoorganización de los sistemas desplazó a la morfogénesis y retroalimentación positiva con que estaba asociado el enfoque sistémico más tradicional (Arnold, Urquiza y Thumala, 2011). En tal sentido, una de las principales innovaciones fue el surgimiento del término autopoiesis, princip de Humberto Maturana y Francisco Varela (1995) el que dice relación con la capacidad de los sistemas para autoproducir recursivamente sus propios elementos, de tal manera que el sistema no tenga que atender a los recursos que le brinda su entorno.

En base a las inquietudes que suscitó la implementación de la autopoiesis en la Teoría de los Sistemas Sociales, Luhmann se vio enfrentado a la necesidad de generar una apropiación efectiva del concepto, para lo cual trasladó su punto de referencia desde un plano biológico a uno comunicativo. Por lo mismo, al momento de considerar las bases del programa autopoiético luhmanniano, este requiere despojarse del sesgo que atiende solo al metabolismo celular proyectándose hacia los eventos comunicativos con proyectandose hacia los eventos com (Vinicativos con que operan long, 2008)

Frente a estos planteamientos, el Trabajo Social no quedó indiferente. En gran medida, esto se vio reflejado en los argumentos de Klaus Harney (1975) sobre el posible vínculo entre esta disciplina y la autor evaluó las potencialidades de los conceptos de sistema y evolución, deteniéndose particularmente en cuán atingentes resultaban para el análisis de los Estados de Bienestar y rol del Trabajo Social por aquellos años. A su vez, para enriquecer su esquema de análisis, Harney desarrolló una revisión del funcionalismo de Parsons, para luego contrastar sus principales lineamientos con los que declaraban poseer los mayores representantes de la escuela de Frankfurt. No obstante, debido a las propias orientaciones del Trabajo Social, y a su subordinación frente a la tecnología, el estudio de Harney derivó en una serie de estandarizaciones y criterios uniformes, sin mayor detalle ni profundización.
Enlace convergente 11: el Trabajo Social como sistema funcional

A la luz de los argumentos presentados con anterioridad respecto al Trabajo Social y sus primeros acercamientos con la Teoría de los Sistemas Sociales, nos inclinamos por sostener que tal disciplina se alza mediante una estructura operativa que nos permite reconocerla como un sistema funcional de la sociedad moderna. En tal sentido, consideramos que desde su abordaje como un sistema que despliega operaciones altamente especializadas, Trabajo Social cumple con el requisito elemental de los sistemas sociales que precisa Luhmann: operar en base a las comunicaciones. Ahora bien, mediante esta premisa nos interesa establecer los siguientes alcances:

En base a la encarnizada complejidad de la sociedad moderna (Luhmann, 1998), y a las premisas indicadas anteriormente respecto a los lineamientos axiales de la Teoría de los Sistemas Sociales de Niklas Luhmann, el Trabajo Social puede ser entendido como un sistema funcional que despliega operaciones ligadas a escenificar opciones de reinclusión para domiciliaridades sociales dañadas, es decir, para aquellas que no tienen acceso a las prestaciones de los sistemas funcionales elementales, que en palabras de Robles (2006b), corresponden a una exclusión primaria (por ejemplo, el estar excluido de un círculo familiar). A partir de lo anterior el Trabajo Social se orienta a impulsar condiciones auspiciosas para la forma persona en las comunicaciones de la sociedad (Robles, 2002), mediante lo cual reconfigura el esquema de la inclusión/exclusión en la medida en que revela la falsa igualdad de oportunidades que pregona la sociedad moderna. De allí que el hecho de extender alternativas de inclusión en la sociedad moderna deba contemplar que la diferenciación funcional se encarga de neutralizar su factibilidad de realización o de reducir su probabilidad.

No obstante, el Trabajo Social no puede supone maximizar las opciones de reinclusión desde un criterio transversal a la sociedad moderna, debido que ningún sistema funcional está en condiciones de poseer un acceso privilegiado a la realidad o de colonizar al resto. Entonces, la gran cruzada que debe contemplar el Trabajo Social es la de producir condiciones de posibilidad para la inclusión, es decir, operar funcionalmente para que la forma persona logre configurarse como un domicilio socia estituido en base a la inclusión (Robles, 2006b). Si perjuicio de lo anterior, para lograr esto es necesario que el Trabajo Social intervenga en las estructuras 
de comunicación que inciden en la reconstrucción de las domiciliaridades sociales a partir de dos ejes neficiario (individual o colectivo, o ambos) que es resignificado bajo el rótulo de caso, y b) intervenir cializados del Traba nclusión de dichos beneficiarios (casos).

En el primer eje, la idea principal corresponde a que los(as) trabajadores(as) sociales se disciplinan con la finalidad de estar constantemente renovando la imagen del (de la) beneficiario(a) al (a la) que deben esto, identificarlo(a) como un caso viable de intervenir en cuanto a la reconstrucción de su domicilio social dañado mediante estructuras comunicativas que fomenten sus condiciones de posibilidad de inclusión. Mientras que en el segundo eje, se tematiza el modo en que dichos(as) trabajadores(as) sociales procesan el contexto de los(as) beneficiarios(as) entendidos como casos- para logar insertarse en los programas que regulan su inclusión en los sistemas funcionales más elementales.

Por intermedio de lo anterior, nos parece atingente mencionar que el Trabajo Social -en gran medidase alza como un sistema funcional especializado en tanto despliega estrategias innovadoras de contro sobre el metacódigo de la sociedad moderna: inclusión/excl de los dominios sistémicos del Trabajo Social, debe recurrir necesariamente a otros dos códigos que e son inherentes a esta disciplina: caso/no-caso ayuda/no-ayuda (Fuchs, 2000, citado en Robles 2002). Ambos recursos operativos -según indica Fuchs- están enfocados en actualizar el esquema de ayuda de los que no. Esto brinda la posibilidad de reconocer domicilios sociales dañados altamente parecidos en términos de su constitución, pero disímiles en sus disposiciones contextuales. Sin embargo, tal criterio genera una discriminación positiva, lo que ratifica que se privilegien los casos mas ciones de sobrellevar la exclusión.

No obstante, Baecker (1994) da cuenta de una postura muy distinta. En tal sentido, el autor señala que el Trabajo Social y la Teoría de los Sistemas Sociales se encuentran en un plano de tensiones, ya que ambos polos están distanciados desde momento en que no comparten una visión objetivista acerca del molo en que son constiulos fundamentales: a) actualizar la existencia de un be cializadas del Trabajo Social en función de regular clusión/exclusión. No obstante, cualquier esfuerz y/o atisbo por incidir en tal esquema binario desde inclusion/exclusion, a ráz de lo cul, el Trajo Social logra distinguir entre los casos que requieren perjudicados por encima de los que están en condi- los problemas sociales, limitándose únicamente a intercambiar comunicaciones sobre el análisis que hace cada uno sobre los medios de comunicación y la política. De este modo, se extiende que dicho vínculo no puede suponer una mejor comprensión de las turbutencis en la sociedal, ni menos, el dede las turbulencias en la sociedad, ni menos, el desarrollo de estrategias que mermen la exclusión de los sistemas funcionales.

A pesar de esto, son más numerosos los argumentos a favor del vínculo. De hecho, Scherr (2002) comenta que desde la visión del Trabajo Social, se ha constatado un gran vuelco sobre el concepto de comunicación y el modo en que esta disciplina pondría énfasis en la estructura de los eventos de comunicación. Por ende, el autor recalca la importancia que tiene la Teoría de los Sistemas Sociales en la mayor riqueza operativa del Trabajo Social, al igual que pone de manifiesto cuán provechoso sería generar más iniciativas como esta. Sobre lo mismo, "los conceptos acoplamiento y acoplamiento estructural, son de vital importancia para entender la relación de esta disciplina y la teoría que Luhmann aporta" (Scherr, 2002, p. 267). De manera cercana, Hollstein-Brinkmann (2006) elabora una revisión de la influencia que ha considerado la Teoría de Sistemas y sus vertientes en la discusión actual sobre el Trabajo Social en Alemania. De esta forma, identifica algunos alcances desde el Constructivismo Radical de Maturana y Varela, las exploraciones de los Sistemas Ecológicos de Bertallanffy, y por último, la influencia de la Teoría de Sistemas Sociales de Niklas Luhmann.

A su vez, Fuchs (2003) sostiene que el andamiaje del Trabajo Social está alineado con la idea de restituir las oportunidades de inclusión mediante el fomento de canales de ayuda, lo que en gran medida se traduce en actividades que pretenden relegar las amenazas que la sociedad contemporánea despliega recursivamente en contra de aquellos(as) más susceptibles a las arremetidas sociales, políticas, económicas, entre muchas otras. En este sentido, dicha modalidad de asistencia comprende una interfaz funcional, la que en términos concretos, se orienta a visibilizar los esquemas morales y éticos que se circunscriben a las labores desempeñadas por el Trabajo Social, especialmente, en cuanto a las dinámicas de intervención. Ahora bien, en este punto es necesario subrayar que dichas actividades están mediadas por un respaldo profesional que determina cómo, dónde y cuándo es pertinente desplegar un asesoramiento contextualizado que oriente la intervención. Por su parte, Bommes \& Scherr (2000) consideran al Trabajo Social como un método específico de comunicación, orientado principalmente a identificar las razones que hacen necesario y legítimo el brindar ayuda, las que en última instancia, apuntan a las regulaciones legales que delimitan estas nociones, el Trabajo Social se enarbola como un rendimiento operativo que presta asistencia en la conformación de las "reglas" inherentes a la sociedad, utilizando para esto los recursos sociales y políticos que están a su alcance.

Desde esta óptica, los componentes ético-políticos que guían el devenir profesional del Trabajo Social, en cómo son tematizados los domicilios sociales dañados en la actualidad. En términos concretos, esto se traduce en aquellos casos que son identificados como los beneficiarios de estrategias paliativas frente a la exclusión. Un claro ejemplo de esto se encuentra en los casos que requieren de tratamientos frente al consumo de drogas, iniciativas de reinserción social posteriormente a una condena afectiva, orientaciones que mermen la deserción escolar, talleres de prevención acerca de la violencia intrafamiliar, ente otros.

En cuanto a los argumentos de Wagner (2006), el Trabajo Social da cuenta de los problemas y desafíos estructurales más apremiantes en el devenir de la sociedad actual, frente a un panorama de recursos entender a ste ulimo desce trajo Social, debe entender a este último como un dominio complejo de acción, donde la teoría y la práctica se concatenan para ofrecer posibilidades de subsanación y reparación de biografías personales que han sido truncadas y/o empañadas reiteradamente.

Paralelamente, Maaß (2007) asegura que los factores más gravitantes en el Trabajo Social contemporáneo apuntan al modo en que la dispersión efectiva de sus ámbitos de comunicación permite generar rutas de acceso más expedito a contextos de inclusión, o en su defecto, expandir las posibilidades de simular oportunidades de reinclusión. Así, estos procedimientos se justifican mediante el recurso de la prevención, es decir, gracias al hecho de mermar las condiciones de exclusión y de compensar los fallos funcionales de otros sistemas. De manera cercana, Mayrhofer (2009) rescata lo que a su parecer corresponde a uno de los mayores beneficios que las atribuciones sistémicas plasman En este punto, nuestra alusión a la disposiciones operativas del código empoderar/no-empoderar se respaldan en las distinciones satisfagan de inzamos como aulores. No obstante, esto no involucra que nos cerremos a la idea de que existan otros códigos que satisfagan de mejor manera los requerimientos del Trabajo Social contemporáneo, y por ende, del código caso/no-caso.

sobre el Trabajo Social: hacer visible la pobreza, la concentración de la riqueza, y por sobre todo, la desigualdad. En tal sintonía, la (re)producción de a desigualdad supone una comunicación basada en la permanente actualización de los criterios de exclusión que forjan la diferenciación funcional de la sociedad moderna, frente a lo cual el Trabajo Social incrementa la oferta de inclusión para estabilizar dicho fenómeno. pertinente destacar la investigación de Camelo Cifuentes (2007), quienes establecen un abordae sobre la discusión epistemológica en el Trabajo Social. En tal sintonía, ambas se inclinan por mencionar que a partir de la crisis de los metarelatos, la perspectiva sistémica-luhmanniana se alza como el recurso más idóneo para observar la complejidad de as problemáticas sociales en la actualidad. A grandes rasgos, se menciona que "el concepto de sistema posibilita visualizar convergencias, más que disyunciones o antagonismos. Lo que en primera instancia se puede evidenciar en un sistema, es la presencia de lo diverso y de lo diferente; todo sistema es uno y múltiple" (Garciandía, 2005, p. 123 en Camelo y Cifuentes, 2007)

En este punto, creemos importante extender una inflexión en torno a la visión del Trabajo Social como sistema funcional: es necesario considerar la viabilidad de reemplazar el código ayuda/no-ayuda por el de empoderar/no-empoderar ${ }^{4}$. Con esto, nos nteresa hacer eco de las formas de intervenció que el Trabajo Social lleva a cabo en la actualidad, especialmente, desde aquellas que apuntan a evitar promover una visión filantrópica y asistencialista de la disciplina, como lo propuesto por Faleiros (2003) y recogido en las investigaciones de Mauus (1999). Por lo mismo, esta nueva orientación pretende emplazar al Trabajo Social en torno a las bases de una corriente movilizadora que no pretende ayudar a los(as) beneficiarios(as), sino que busca empoderarlos(as) -ratificando el distanciamiento de una visión asistencialista- para que logren acceder a las posibilidades de su inclusión en las prestaciones más auspiciosas de los sistemas funcionales que constituyen la sociedad moderna.

Lo cierto es que nuestra principal intención responde, a través de lo enunciado en los párrafo anteriores, a tomar como marco de referencia lo planteado por Teresa Matus acerca de los funda-
Ahora bien, desde el contexto latinoamericano es 
mentos del Trabajo Social contemporáneo, haciendo hincapié en las precisiones que dicha contribución nos brinda para aproximar la disciplina a la Teoría de los Sistemas Sociales de Niklas Luhmann. A raíz de esto, el principal desafío al que nos hemos enfrentamos dice relac mientas conceptuales que nos brinda la Teoría de los Sistemas Sociales para elaborar un esquema de observación sistémica acerca del Trabajo Social contemporáneo.

Por lo mismo, nos hemos remitido particularmente a Robles (2002) y Matus (1999) para vislumbrar que en la soci trabajadores(as) sociales adolece de un menoscabo que se centra en subrayar cómo sus metodologías están ligadas únicamente con el hecho de realizar diagnósticos tangenciales acerca de los(as) beneficiarios(as) que son identificados como casos a intervenir. De allí que en torno a la formación académica del Trabajo Social se observe un déficit de autodescripc municativa que avale un recurso operativo que llegue a menguar la exclusión (la probable y acumulativa) en torno a lo sistemas funcionales de la sociedad moderna.

No obstante, resulta innegable que tal escenario de detrimento hacia la disciplina genera un reduccionismo sobre el modo en que esta logra operar por sobre la complejidad estructural de donde emana la desigualad social. En consecuencia, no se le brinda suficiente relevancia a la posibilidad de que el Trabajo Social intervenga en los domicilios sociales dañados, con énfasis en que estos lleguen a ser tratados como un perfil auspicioso de la forma persona. A pesar de esto sería antojadizo mencionar que forma persona pueda ser modificada sin inconvenientes por el sistema del Trabajo Social, sino que es necesario recordar que este último debe proceder estrictamente en función de los eventos comunicativos que excluyen a los domicilios sociales.

\section{Reflexiones finales}

En base a lo mencionado hasta este punto, nos inclinamos por indicar algunos alcances sobre la naturaleza de la propuesta que desarrollamos. Al respecto, resulta interesante dejar planteadas interrogantes para futuras investigaciones, tales como ¿cuáles son los eventos operativos que se generan al afirmar que el Trabajo Social es un sistema funcional? En este sentido, tenemos la claridad de que comunicativos más elementales, entonces ¿es fac- tible postular que para esto necesita recurrir a la intervención social?, o en tras palabras, ¿la comunicación de la intervención social permite hablar de la autopoiesis del Trabajo Social como sistema funcional?

De manera similar, nos genera inquietud el poder vislumbrar cuál es el código más atingente y los programas más inherentes al operar exclusivo del Trabajo Social. A pesar de esto, hemos propuesto que el código ayuda/no-ayuda puede llegar a ser reemplazado por el de empoderar/no-empoderar, sin embargo, ¿cuáles vendrían a ser los programas de normas y reglas tácitas regularían aquel código en términos de la inclusión y la exclusión?

Tales preguntas, a pesar de erigirse desde la Teoría de los Sistemas Sociales, no se alejan de los cuestionamientos más clásicos del propio Trabajo Social. De hecho, tales interrogantes se encuentran imbricadas en torno a los fundamentos epistemológicos de la disciplina y a los marcos interpretativos que esta utiliza para fundamentar su quehacer académico y profesional. Uno de los alcances que se ha encargado de dar cabida a tales problemáticas, dice relación con la propuesta polifónica de Matus (1999), quien plantea que el Trabajo Social ha tomado prestados elementos de diversas ramas de las Ciencias Sociales - inclusive, de las ciencias duras (escuelas de corte tecnológico)- para elaborar esquemas explicativos de las situaciones que genera el leitmotiv de la disciplina. A partir de lo anterior, no parecería tan distante el poder aunar esfuerzos por seguir ahondado en tales planteamientos desde lo que hemos propuesto como una aproximación hacia el Trabajo Social desde las observaciones de la Teoría de los Sistemas Sociales.

En efecto, esto se podría ver reflejado en cómo, desde una aproximación sistémica, sería factible llevar a cabo futuras investigaciones sobre conceptualizaciones que utiliza el Trabajo Social contemporáneo, como son: la vulnerabilidad, la línea de la pobreza, las personas en situación de calle y otras formas de nombrar problemáticas ligadas directamente al esquema de la inclusión/exclusión que oficia la Teoría desarrollada por Luhmann. Lo anterior encuentra gran asidero en la medida en que se reconoce a la sociedad contemporánea como fomentadora mayoritaria de la desigualdad, la que se consagra por la afirmación de que todos(as) están determinados(as) inexorablemente a quedar incluido(as) o excluido(as) en algún evento y/o circunstancia a lo largo de sus biografías personales.

\section{Referencias bibliográficas}

ARNOLD, M., URQUIZA, A., THUMALA, D. (2011). Recepción del concepto de autopoiesis en las ciencias Sociales. Revista Sociológica, 26, 73, 87-108.

ANDER-EGG, E. (1984). El desafio de la reconceptualización. Alicante: Humanitas

BAECKER, D. (1994). Soziale Hilfe als Funktionssystem der Gesellschaft. Zeitschrift für Soziologie, 23, 2, 93-110.

BERTALANFFY, L. VON (1968). General System theory: Foundations, Development, Applications. New York: George Braziller.

BOMMES, M. \& SCHERR, A. (2000). Soziologie der Sozialen Arbeit. Eine Einführung in Formen und Funktionen organisierter Hilfe. Weinheim: Juventa.

BRAECKMAN, A. (2006). Niklas Luhmann's systems theoretical redescription of the inclusion/exclusion debate. Philosophy Social Criticism, 32, 1, 65-88.

CAMELO, A. Y CIFUENTES, R. (2007). Contextualización de la discusión epistemológica de metodología integrada. Revista Tendencia \& Retos, 12, 137-155.

FALEIROS, V. (1970). Metodología e ideología del Trabajo Social. Miraflores Perú: CELATS

FALEIROS, V. (2003). Estrategias de empowerment en Trabajo Social. Buenos Aires: Lumen.

FUSCHS, P. (2003). Die Moral des Systems Sozialer Arbeit - systematisch. Consultado el 1 de noviemrbre de 2012 desde http://www.sozialarbeit.ch/dokumente/ethik.pdf

HARNEY, K. (1975). Sozialartbeit als System, Die entwicklung de systembegriff durch $\mathrm{N}$. Luhmann im hinblick auf eine Funktionsbestimmung sozialer arbeit. Zeitschrift für Soziologie, 4, 2, 103-114.

HEALY, K. (2001). Trabajo Social: Perspectivas contemporáneas. Madrid: Morata.

HOLLSTEIN-BRINKMANN, H. (2006). Systems theories a comparison. What contribution do systems theories make to social work?. Consultado el 2 de noviembre de 2012, desde http://profs.efh-darmstadt.de/fileadmin/uploads/ users/Hollstein-Brinkmann/Systems_Theory_A_Comparison.pdf

LASZLO, A. \& KRIPPNER, S. (1998). Systems Theories: Their Origins, Foundations, and Development. In J.S Jordad (Ed), Systems Theorles and A Priori Aspects of Per-

LUHMANN, N. (1992). Teoría de la Sociedad. México: Universidad de Guadalajara.

LUHMANN, N. (1996). Introducción a la Teoría de Sistemas. México: Anthropos.

LUHMANN, N. (1997). Observaciones de la modernidad. Barcelona: Paidós.

LuHMANN, N. (1998). Complejidad y Modernidad. De la unidad a la diferencia. Madrid: Trotta.
LuHMANN, N. (2007). La sociedad de la sociedad. México: Herder

MAAß, O. (2007). Die Soziale Arbeit als Funktionssystem der Gesellschaft? - Eine systemtheoretische Analyse.
Consultado el 1 de noviembre de 2012, desde http:// www.db-thueringen.de/servlets/DerivateServlet/Derivate-12856/Dissertation.pd

MAKAROVIĈ, M. (2001). Some Problems in Luhmann's Social Systems Theory: Differentiation, Integration and Planning. Družboslovne razprave, 17, 32, 59-70.

MATURANA, H. y VARELA, F. (1995). De máquinas y seres vivos. "Autopoiesis": la organización de lo vivo. Santiago: Universitaria

MATUS, T. (1999). Propuestas contemporáneas en Trabajo Social, hacia una intervención Polifónica. Buenos Aires: Espacio.

MATUS, T. (2003). La intervención social como gramática. Hacia una semántica propositiva del Trabajo Socia frente a los desafíos de la globalización. Revista de Tra-

MAYRHOFER, H. (2009). Soziale Inklusion und Exklusion: (system-) theoretische Unterscheidung als Beobanoviembre de 2012 deste hup trinder de 2012, desapital/article/viewFile/108/145. pd

MINUCHIN, S. y CHARLES, F. (1984). Técnicas de terapia familiar. Barcelona: Paidós.

PARSONS, T. (1988). El Sistema Social. Madrid: Alianza.

QUEZADA M. et al, (2002). Perspectivas metodológicas en Trabajo Social. Buenos Aires: Espacio

ROBLES, F. (2002). Opciones de reinclusión para domiciliaridades dañadas. Cuaderno Venezolano de Sociología, $11,1,9-24$.

ROBLES, F. (2004). Sistemas de Interacción, Doble Contingencia y Autpoiesis Indexical. Cinta de Moebio, 15.

ROBLES, F. (2005). Contramodernidad y Desigualdad Social: Individualización e individuación, inclusión/ de una sociología de la exclusión. Revista MAD, 12

ROBLES, F. (2006a). El futuro como riesgo y la victori de la contingencia: Entrelazando senderos disociadosdesocultando paradojas de la modernidad. Sociedad Hoy, Primer Semestre, 10, 15-39.

ROBLES, F. (2006b). Autopoiesis, inclusión y tiempo. La indolencia ante la exclusión social. Revista de la Academia, Segundo Semestre, 11, 91-106.

ROBLES, F. (2010). Los ecos de la vergüenza: Pasado y presente de la exclusión social en Chile. Consultado el 9 de agosto de 2012, desde http://es.scribd.com doc/44343702/Los-Ecos-de-La-Verguenza 
SCHERR, A. (2002). Soziologische Systemtheorie ats Grundlage einer Theorie der Soziaten Arbeit. Consultado el 1 de noviembre de 2012, desde http://w3mediapool $\mathrm{hm}$.edu/mediapool/media//k11/k11_lokal/forschungpublikationen/lehrmaterialen/dokumente_112/ sagebiel_1/Scherr-2002 SozSystemtheorie_als_Grundage_einer_Theorie_d_SA-Luhmann.pdf

VEMON, D. \& FURLONG, D. (2008). Relativistic Ontologies, Self-Organization, Autopoiesis, and Articial Life. A Progression in the Science of the Autonomous. Essay 4 Part II: A Scientic Development. Dublin: Trinity College Press.

WAGNER, T. (2006). Die Soziale Arbeit der Sozialen Arbeit? - Ein kurzer Blick auf die (Selbst-) Beobachtung eines 2012 systems. Consultado el 1 de noviembre de soziale_arbeit_der_sozialen_arbeit.pdf

\section{Representaciones sociales del VIH-SIDA en la Región del Maule, Chile}

Social representations of HIV/AIDS in the Maule Region, Chile

Óscar Labra, Ph.D

Profesor Departamento de desarrollo humano y social. Universidad de Quebec en Abitibi-Témiscamingue 445, boulevard de l'Université. Rouyn-Noranda (Quebec) J9X5E4. Teléfono: 819-762-0971 (2335). Fax: 819-797-4727.Correo: oscar.labra@uqat.ca Serge Dumont Ph.D.

Director Cientifico CSSS de la Vieille-Capitale, 880, rue Père-Marquette, 3er piso Quebec (Quebec) G1S 2A4. Teléfono: 418-681-8787, poste 3964. Fax: 418 687-9694. Correo: serge.dumont@svs.ulaval.ca

\section{Resumen}

El objetivo de la presente investigación consistió en producir una descripción de las representaciones sociales del VIH-SIDA tal cual son culturalmente construidas y compartidas al interio de los Servicios de Salud de la Región del Maule, Chile. Esta investigación cualitativa, demuestra que las representaciones sociales del VIH-SIDA en el Maule se elaboran y se estructuran bajo la influencia de procesos socioculturales en el seno comunitario y que las representaciones sigue siendo perjudiciales para las personas que viven con el VIH-SIDA (PWIH-SIDA). No obstante, el discurso de los profesionales de la salud y los pacientes revela cambios positivos observados en los equipos de cuidados de primera linea, donde la práctica médica en esta población vulnerable seria más valorizada y menos estigmatizada. Los que reciben atención en salud manifiestan sentirse bien acogidos y escuchados. Las actitudes hacia ellos serian más respetuosas de parte de los profesionales de los equipos en VIH del Maule dadas las distintas formas de sufrimiento que las PWIH-SIDA vivencian cotidianamente.

Palabras clave. representaciones sociales, profesionales de la salud, PWIH-SIDA.

Abstract

The aim of the study is to provide an overview of social representations of HIV/AIDS as they are culturally constructed and shared within the social and health services environment in centra Chile's Maule Region. This qualitative study demonstrates that sociocultural processes influence the elaboration and structuring of social representations of HIV/AIDS in Maule Region communities and that those representations continue to be prejudicial towards people living with HIV/AIDS (PLHIV/AIDS). However, the testimonies of health professionals and patients alike, reveal positive changes among first line health care personnel that have lead to the increased valorization and reduced stigmatization of care delivered to this vulnerable population. People receiving some care for HIVIAIDS in their health express some feelings of being understood and comforted. Attitudes forwat these people wouldo more respectul from professionat of the HIV team at the Mate given the differents ways of suffering that PWIH-SIDA live commonly.

Key words. Social representation, health proffesionals, PLHIV/AIDS.

\section{Introducción}

No hay duda de que el VIH-SIDA constituye un verdadero problema social en Chile, como en otras partes del mundo. Ahora bien, la construcción simbólica de la pandemia ha afectado no solo la vida de las PVVIH-SIDA sino también la de los equipos en VIH. Los estudios que se interesaron específicamente sobre la relación entre pacientes VIH y equipos te $\mathrm{VIH}$ lo muestran bien (Goodwin et at. 2003; Curtis y otros, 2000; Watney, 1989). La estigmati- zación y la discriminación favorecen el aislamiento comprometen el apoyo social hacia ellos. Además, la exposición pública de la seropositividad de las PVVIH-SIDA es perjudicial para la mantención de empleo y crea obstáculos en la atención de cuidados en salud (Chan et al. 2008; Teixeira et Silva 2008; Varas-Díaz et Marzán-Rodríguez, 2007; Chan et Reidpath, 2007; Kinsler et al. 2007; Fiscella et al. 2004). La litera discriminatoras basadas en prejuicios referen a 